\title{
Expression of the AT2 receptor developmentally programs extracellular signal-regulated kinase activity and influences fetal vascular growth
}

\author{
Masahiro Akishita, Masaaki Ito, Jukka Y.A. Lehtonen, Laurent Daviet, Victor J. Dzau, \\ and Masatsugu Horiuchi
}

Cardiovascular Research, Department of Medicine, Brigham and Women's Hospital, Harvard Medical School, Boston, Massachusetts 02115, USA

Address correspondence to: Masatsugu Horiuchi, Cardiovascular Research, Department of Medicine, Brigham and Women's Hospital, Harvard Medical School, 75 Francis Street, Thorn-13, Boston, Massachusetts 02115, USA.

Phone: (617) 732-8917; Fax: (617) 975-0995; E-mail: mhoriuchi@rics.bwh.harvard.edu

Received for publication September 10, 1998, and accepted in revised form November 10, 1998.

\begin{abstract}
Angiotensin II type 2 (AT2) receptor is abundantly expressed in vascular smooth muscle cells (VSMC) of the fetal vasculature during late gestation (embryonic day 15-20), during which the blood vessels undergo remodeling. To examine directly the influence of AT2 receptor expression in the developmental biology of VSMC, we studied cultures of VSMC from fetal and postnatal wild-type (Agtr2 $\left.2^{+}\right)$and AT2 receptor null (Agtr2 $\left.{ }^{-}\right)$mice. Consistent with in vivo data, AT2 receptor binding in cultured Agtr $2^{+}$VSMC increased by age, peaking at embryonic day 20, and decreased dramatically after birth. Angiotensin II-induced growth in Agtr2 ${ }^{+}$VSMC (embryonic day 20) was increased by the AT2 receptor blocker PD123319, indicating that the AT2 receptors are functional and exert an antigrowth effect in $A g t r 2^{+}$ VSMC. Growth of VSMC in response to serum decreased age dependently and was higher in Agtr2- than in $A g t r 2^{+}$, inversely correlating with AT2 receptor expression. However, serum-induced growth in $A g t r 2^{+}$ and Agtr2- VSMC and the exaggerated Agtr2- VSMC growth was maintained even in the presence of PD123319 or losartan, an AT1 receptor blocker. Moreover, Agtr2- VSMC showed greater growth responses to platelet-derived growth factor and basic fibroblast growth factor, indicating that Agtr2- cells exhibit a generalized exaggerated growth phenotype. We studied the mechanism responsible for this phenotype and observed that extracellular signal-regulated kinase (ERK) activity was higher in Agtr2- VSMC at baseline and also in response to serum. ERK kinase inhibitor PD98059 inhibited both growth and ERK phosphorylation dose-dependently, while the regression lines between growth and ERK phosphorylation were identical in Agtr $2^{+}$and Agtr2 $2^{-}$VSMC, suggesting that increased ERK activity in Agtr2VSMC is pivotal in the growth enhancement. Furthermore, the difference in ERK phosphorylation between $\mathrm{Agtr2} 2^{+}$and $\mathrm{Agtr} 2^{-}$was abolished by vanadate but not by okadaic acid, implicating tyrosine phosphatase in the difference in ERK activity. These results suggest that the AT2 receptor expression during the fetal vasculogenesis influences the growth phenotype of VSMC via the modulation of ERK cascade.

J. Clin. Invest. 103:63-71 (1999).
\end{abstract}

\section{Introduction}

Angiotensin II (Ang II), a key regulator of cardiovascular homeostasis, exerts various actions in its diverse target tissues that control vascular tone, hormone secretion, tissue growth, and neuronal activity (1). To date, at least two Ang II receptor subtypes are defined: type 1 (AT1) and type 2 (AT2). Most of the actions of Ang II are mediated by the well-characterized AT1 receptor, while less is known about the functions of the recently cloned AT2 receptor $(2,3)$.

In vitro studies have shown that the AT2 receptor mediates growth inhibition in vascular smooth muscle cells (VSMC) (4, 5), coronary endothelial cells (6), PC12W cells, which is a rat pheochromocytoma cell line (7), and cardiomyocytes (8). It mediates differentiation in neuronal cells $(7,9)$ and/or apoptosis in vascular smooth muscle cells (VSMC) (10), PC12W cells $(11,12)$, cardiomyocytes (13), R3T3 mouse fibroblasts $(11,14)$, and endothelial cells (15). Signal transduction pathways of these AT2 receptor actions include extracellular signal-regulated kinase (ERK)-1 and -2, also known as p44 and p42 mitogen-activated protein (MAP) kinases, which are critical to cell proliferation, differentiation, and, in some cells, hypertrophy (16). AT2 receptor stimulation causes the inactivation of ERK, which may be mediated by the activation of protein tyrosine phosphatases such as SHP-1 (17) and MAP kinase phosphatase-1 (MKP-1) $(11,12)$.

The AT2 receptor is abundantly and widely expressed in fetal tissues, but is present only at low levels in adult tissues, including tissues of the adrenal gland, brain, heart, uterine myometrium, and atretic ovarian follicles $(18,19)$. The AT2 receptor expression is upregulated in some disease states such as vascular injury $(4,20)$, myocardial infarction (21), and failing heart (22). The AT2 receptor expression in the aorta also exhibits a developmental pattern in that it is very low (or undetectable) during early embryonic development, but is very high during the later stages of embryonic development and in the neonate (23, 
AT1 receptor binding

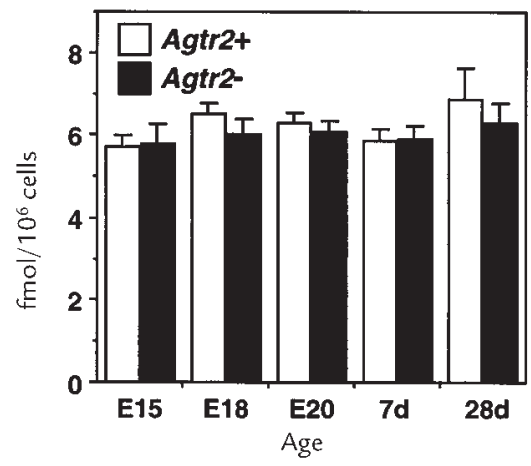

AT2 receptor binding

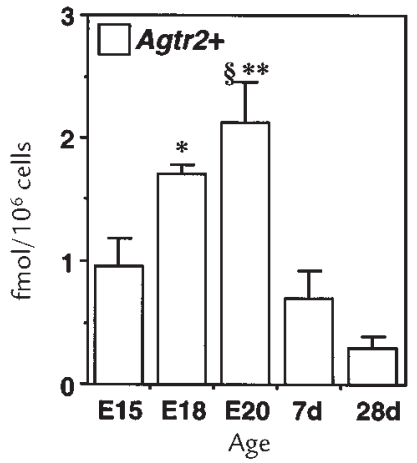

Figure 1

AT1 and AT2 receptor binding densities in wild-type $\left(\right.$ Agtr2 $\left.{ }^{+}\right)$and AT2 receptor null (Agtr2-) VSMC. Radioligand binding assay was performed using subconfluent Agtr2 ${ }^{+}$and Agtr2- VSMC derived from embryonic day 15 (E15), day 18 (E18), and day 20 (E20) fetuses, and from mice of postnatal day $7(7 d)$ and day $28(28 d)$. Similar results were obtained in at least three different culture lines. The values are expressed as mean \pm SEM $(n=$ 4). $\S P<0.05$ vs. E15; ${ }^{*},{ }^{*} P<0.05,0.01$ vs. $28 \mathrm{~d}$. AT1, angiotensin II type 1 ; AT2, angiotensin II type 2; VSMC, vascular smooth muscle cells.

24). After birth, the level of this receptor declines rapidly. The transient expression of the AT2 receptor suggests that it plays a role in fetal vascular development, growth, and/or differentiation. Indeed, in utero administration of AT2 blocker could inhibit the developmental reduction of the DNA synthesis in fetal rat aorta (4), supporting the notion that this receptor is implicated in vasculogenesis.

In this study, to further investigate the role of the AT2 receptor in the developmental biology of VSMC, we studied cultures of VSMC from wild-type $\left(A g t r 2^{+}\right)$and AT2 receptor null (Agtr2 $\left.{ }^{-}\right)$mice (25). We analyzed the receptor expression, growth, and signaling in these cells. We demonstrated that Agtr2- VSMC, compared with Agtr2 ${ }^{+}$ VSMC, showed developmentally regulated growth enhancement attributed to a higher ERK activity that is regulated by the tyrosine phosphatase pathway. We postulate that developmental expression of VSMC AT2 receptor programs the subsequent level of ERK activity and influences fetal VSMC growth.

\section{Methods}

Animals. Female mice heterozygous for the AT2 receptor mutant allele (25) were mated with Agtr $2^{+} \mathrm{FVB} / \mathrm{N}$ male mice (The Jackson Laboratory, Bar Harbor, Maine, USA). Pregnant mice were sacrificed by overdose anesthesia, and the fetuses were dissected from uterine decidua. The day when a vaginal plug was observed was considered to be embryonic day 1 (E1). Fetuses at the ages of E15, E18, and E20 were used in this study. In addition, postnatal day 7 and day 28 male mice, which were obtained by the same mating as the fetuses, were used. These fetuses and postnatal mice, backcrossed for six generations into the FVB/N background, had $98 \% \mathrm{FVB} / \mathrm{N}$ and $2 \% 129 / \mathrm{SV}$ background. Animal genotyping was performed as described previously (25), using body or tail genomic DNA samples. The animals were housed in a room where lighting was controlled ( $12 \mathrm{~h}$ on, $12 \mathrm{~h}$ off) and room temperature was kept at $22^{\circ} \mathrm{C}$. They were given standard diet and water ad libitum. All experimental procedures were approved and carried out in accordance with the guidelines of the Harvard Medical Area Standing Committee on Animals.

Culture of VSMC. Thoracic aortae were removed from fetuses and postnatal animals and micro dissected free from the surrounding adventitial tissues. The aortae from individual animals were placed into separate individual test tubes and then digested in collagenase $(1 \mathrm{mg} / \mathrm{ml}$, type II; Sigma Chemical Co., St. Louis, Missouri, USA) in DMEM (Life Technologies Inc., Rockville, Maryland, USA) for $30 \mathrm{~min}$ at $37^{\circ} \mathrm{C}$. After triturating and centrifuging twice, the cells were seeded in $10-\mathrm{cm}$ culture dishes and cultivated in DMEM supplemented with $10 \%$ FBS. Thus, a culture line was isolated from each animal. Culture lines obtained from littermates were maintained exactly in the same manner. Culture medium was changed every other day. At 10-14 days after primary culture, the cells were trypsinized and seeded for the following experiments. Accordingly, all the experiments were performed using the cells at the second passage, except the experiment in which the effect of passages was examined. Comparisons between Agtr2 ${ }^{+}$and Agtr2- cells were performed using littermate culture lines.

In addition to the morphological observation, characterization of VSMC was examined by indirect immunofluorescence using a monoclonal antibody specific for the $\alpha$-smooth muscle actin (clone 1A4; Sigma Chemical Co.) (26).

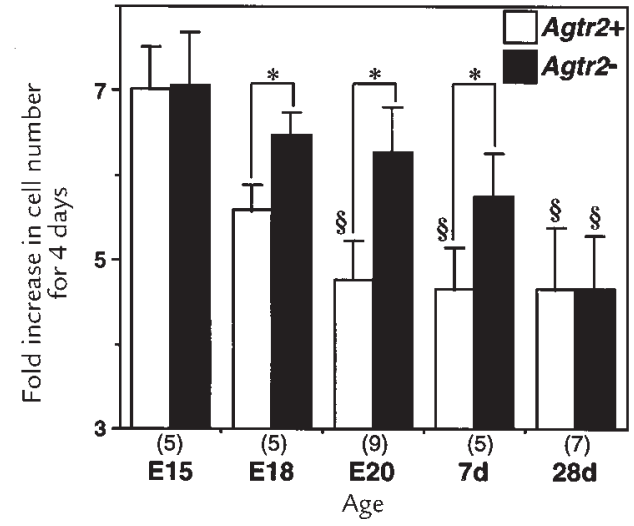

Figure 2

Developmental change in wild-type (Agtr2+) and AT2 receptor null (Agtr2-) VSMC growth. The numbers of VSMC derived from aortae of embryonic day 15 (E15), day 18 (E18), and day 20 (E20) fetuses and from aortae of postnatal day $7(7 d)$ and day $28(28 d)$ mice were counted at 1 day and 5 days after seeding, and then averaged for four wells. The growth of each cell culture line in response to $10 \%$ FBS was expressed as the fold increase in cell number from 1 day to 5 days. The values are expressed as mean \pm SEM of five to nine independent experiments (the number is indicated in parenthesis), each of which consists of several Agtr2 ${ }^{+}$and Agtr2- littermate culture lines. ${ }^{*} P<0.05$ by paired $t$ test; $\S P<0.05$ vs. E15 by Newman-Keuls' test. 
Receptor binding assay. AT1 and AT2 receptor binding was measured using subconfluent cells grown in 24-well plates (Becton Dickinson and Co., Franklin Lakes, New Jersey, USA). After washing twice with PBS containing $0.1 \%$ BSA, the cells were incubated for $1 \mathrm{~h}$ at $37^{\circ} \mathrm{C}$ with $0.2 \mathrm{nM}{ }^{125} \mathrm{I}-\left[\mathrm{Sar}^{1}\right.$,Ile $\left.{ }^{8}\right]$ Ang II (Du Pont Nen Research Products, Boston, Massachusetts, USA) in the absence (for the total count) or presence of $1 \mu \mathrm{M}$ losartan (Merck Research Laboratories, Rahway, New Jersey, USA) or $1 \mu \mathrm{M}$ PD123319 (Research Biochemicals International, Natick, Massachusetts, USA). The cells were then washed twice with ice-cold PBS containing $0.1 \%$ BSA and were lysed in $0.5 \mathrm{~N} \mathrm{NaOH}$. The raw radioactivity count of the lysate was measured by gamma counter. AT1 receptor binding was calculated as the difference between the total count and the count from samples incubated with losartan. AT2 receptor binding was determined by subtracting the count of samples incubated with PD123319 from the total count. The net radioactivity count was converted to molar value by using specific activity of the ligand and was normalized by the cell number, which was measured at the same time.

Developmental change in VSMC growth. To investigate the developmental change in the growth of Agtr2 $2^{+}$and Agtr2- cells, VSMC were seeded in 24-well plates at a density of $3 \times 10^{4}$ cells per well and maintained in DMEM containing 10\% FBS. The medium was replaced every other day. The number of cells were determined using a Coulter Counter (model ZM; Coulter Electronics Ltd., Hialeah, Florida, USA) at 1 day and 5 days after seeding and were averaged for 4 wells. The growth of each culture line was expressed as the fold increase in cell number from 1 day to 5 days. Thus, 5 to 9 independent experiments, each of which consisted of several Agtr2 ${ }^{+}$and Agtr2- littermate culture lines, were performed for each age.

Effects of Ang II receptor blockers on Ang II- or FBS-induced proliferation. VSMC were seeded in 24-well plates at a density of $3 \times$ $10^{4}$ cells per well in DMEM containing 10\% FBS. The following day, the medium was replaced with the defined serum-free (DSF) DMEM containing $0.5 \mu \mathrm{M}$ insulin, $5 \mu \mathrm{g} / \mathrm{ml}$ transferrin, and $0.2 \mathrm{mM}$ ascorbate, and was incubated for $48 \mathrm{~h}$ to induce quiescence. Then the cells were treated for 3 days with DSF containing various agents. For Ang II-induced proliferation, vehicle or Ang II $(0.3 \mu \mathrm{M})$ with or without PD123319 $(10 \mu \mathrm{M})$ or losartan $(10 \mu \mathrm{M})$ was added to DSF. For FBS-induced proliferation, vehicle or 10\% FBS with or without PD123319 (10 $\mu \mathrm{M})$ or losartan $(10 \mu \mathrm{M})$ was added to DSF. Each medium was changed every day. Cell numbers were counted before treatment (baseline) and at 3 days after treatment.

Effects of growth factors on DNA synthesis. DNA synthesis was assayed by measuring ${ }^{3} \mathrm{H}$-thymidine incorporation. VSMC (E20) were seeded in 24-well plates at a density of $5 \times 10^{4}$ cells per well in DMEM containing $10 \%$ FBS. The following day, the medium was replaced with DSF and incubated for $48 \mathrm{~h}$ to induce quiescence. The cells were treated with a DSF-containing vehicle, $10 \%$ FBS, $10 \mathrm{ng} / \mathrm{ml}$ platelet-derived growth factor (PDGF)-BB (Life Technologies Inc.), or $10 \mathrm{ng} / \mathrm{ml}$ basic fibroblast growth factor (bFGF) (Life Technologies Inc.) for $20 \mathrm{~h}$ and were pulsed with 1 $\mu \mathrm{Ci} / \mathrm{ml}^{3} \mathrm{H}$-thymidine (Du Pont Nen Research Products) for an additional $4 \mathrm{~h}$. The cells were washed twice with ice-cold PBS and subsequently incubated with ice-cold 5\% TCA for $20 \mathrm{~min}$ at $4^{\circ} \mathrm{C}$. The cells were washed twice with ice-cold 5\% TCA, then with ice-cold PBS, and were lysed with $0.5 \mathrm{~N} \mathrm{NaOH}$. The radioactivity of the cell lysate was determined using a liquid scintillation counter and was normalized by the cell number.

Determination of ERK activity, phosphorylation, and protein level. Subconfluent Agtr2 ${ }^{+}$and Agtr2- VSMC (E20) were incubated in serum-free DMEM for $16 \mathrm{~h}$ and were treated with $10 \% \mathrm{FBS}$ for 0-30 min. After the medium was completely removed, the cells were washed quickly with HEPES-buffered saline twice and frozen in liquid nitrogen. The cells were lysed in the lysis buffer (25 mM Tris-HCl [pH 7.5], $25 \mathrm{mM} \mathrm{NaCl}, 0.5 \mathrm{mM}$ EGTA, $10 \mathrm{mM}$ $\mathrm{NaF}, 20 \mathrm{mM} \beta$-glycerophosphate, $1 \mathrm{mM} \mathrm{Na}_{3} \mathrm{VO}_{4}, 1 \mathrm{mM}$ PMSF, and $10 \mu \mathrm{g} / \mathrm{ml}$ aprotinin). These lysates were used for kinase assay and immunoblot for phospho-ERK and ERK.

ERK activity was assayed by its ability to phosphorylate myelin basic protein as described previously $(5)$. The lysate $(100 \mu \mathrm{g})$ was precipitated with $5 \mu \mathrm{g}$ of the anti-ERK-1/2 antibody (Upstate Biotechnology, Lake Placid, New York, USA) and a 40- $\mu$ g suspension of protein G Sepharose (Pharmacia Biotech Inc., Piscataway, New Jersey, USA). The immunoprecipitates were incubated for 10 min at room temperature with $24 \mu \mathrm{l}$ of reaction buffer, including $1 \mathrm{mg} / \mathrm{ml}$ myelin basic protein and $40 \mu \mathrm{M} \gamma^{-32} \mathrm{P}$-ATP (Du Pont Nen Research Products) as substrates. The kinase reaction was terminated by adding Laemmli sample buffer. The samples were run on $14 \%$ SDS-PAGE, and the proteins were stained with Coomassie brilliant blue, dried, and analyzed by autoradiography. The bands corresponding to myelin basic protein were cut, and their radioactivity was measured by scintillation counting.

For immunoblotting, the cell lysate was separated on $12 \%$ a

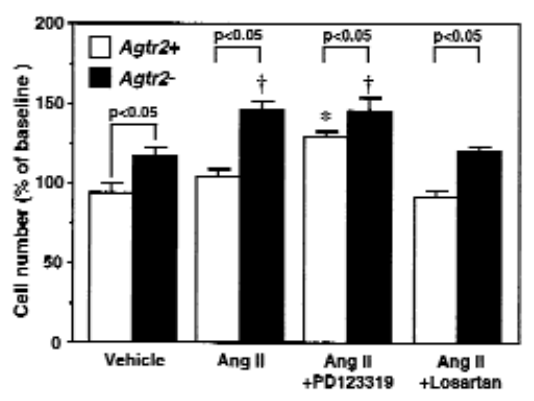

$b$

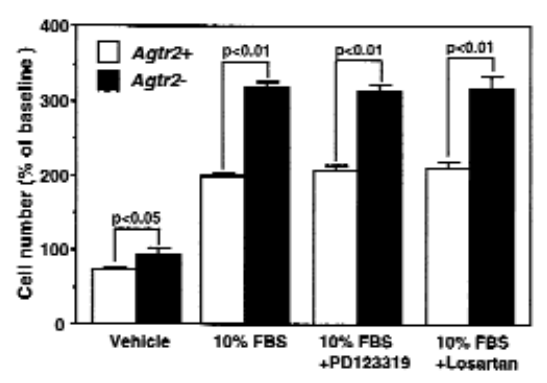

$c$

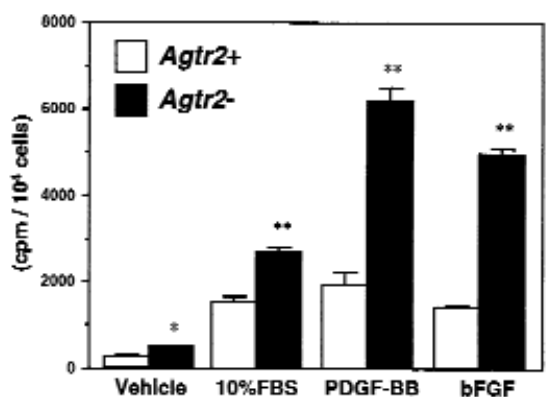

Figure 3

(a-c) Growth of wild-type $\left(\right.$ Agtr2 $\left.{ }^{+}\right)$and AT2 receptor null $($Agtr2-) VSMC derived from the aortae of embryonic day 20 fetuses, in response to angiotensin II $($ Ang II) (a), FBS (b), or other growth factors (c). (a) Subconfluent, quiescent second-passage cells were treated with vehicle, Ang II (0.3 $\mu$ M), Ang II plus PD123319 (10 $\mu \mathrm{M})$, or Ang II plus losartan $(10 \mu \mathrm{M})$ for 3 days. The cell number is expressed as percent of the number before the treatment (baseline). Similar results were obtained in three different culture lines. The values are expressed as mean \pm SEM $(n=6)$. ${ }^{*} P<0.05$ vs. vehicle, Ang II, and Ang II plus losartan; $\nmid P<0.05$ vs. vehicle and Ang II plus losartan. (b) Subconfluent, quiescent second-passage cells were treated with vehicle, $10 \%$ FBS, 10\% FBS plus PD123319 (10 $\mu \mathrm{M})$, or 10\% FBS plus losartan (10 $\mu \mathrm{M})$ for 3 days. The cell number is expressed as percent of the number before the treatment (baseline). Similar results were obtained in three different culture lines. The values are expressed as mean \pm SEM $(n=4)$. $(\boldsymbol{c})$ DNA synthesis was assayed by measuring ${ }^{3} \mathrm{H}$-thymidine incorporation. Subconfluent, quiescent second-passage cells were treated with vehicle, $10 \%$ FBS, PDGF$\mathrm{BB}(10 \mathrm{ng} / \mathrm{ml})$, or bFGF $(10 \mathrm{ng} / \mathrm{ml})$. Similar results were obtained in three different culture lines. The values are expressed as mean \pm SEM $(n=4)$. ${ }^{*},{ }^{*} P<0.05,0.01$ vs. Agtr2 ${ }^{+} . b F G F$, basic fibroblast growth factor; PDGF, platelet-derived growth factor. 

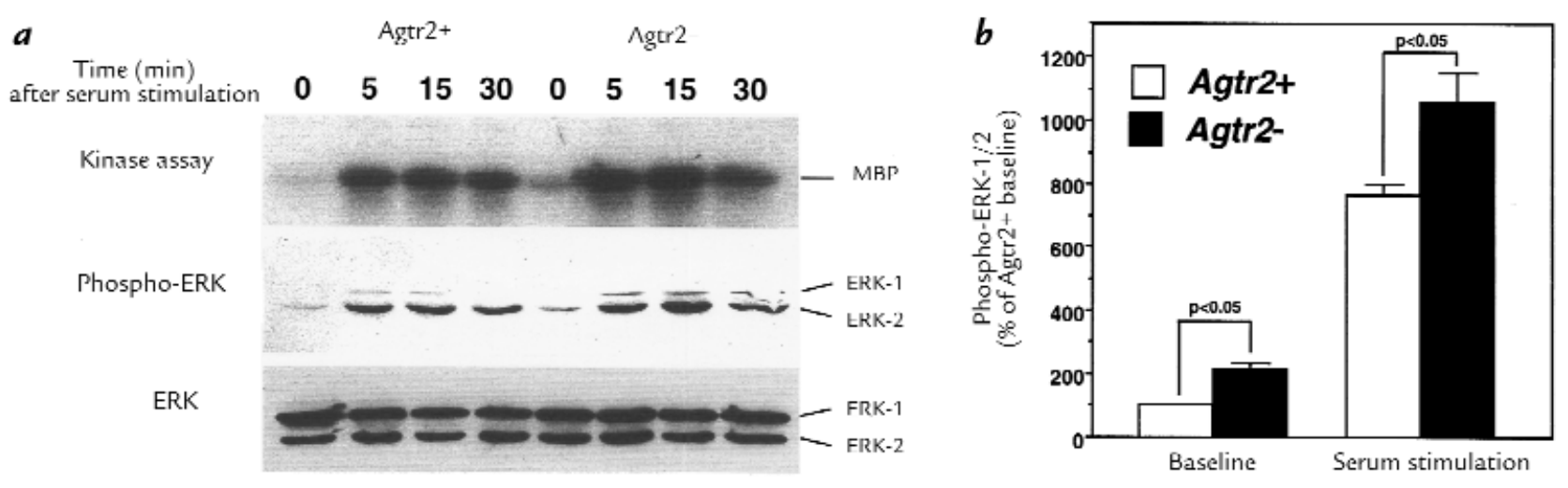

\section{Figure 4}

ERK activity at baseline and in response to serum in wild-type $\left(\right.$ Agtr2 $\left.{ }^{+}\right)$and AT2 receptor null (Agtr2-) VSMC derived from the aortae of embryonic day 20 fetuses. (a) Subconfluent, quiescent second-passage cells were treated with $10 \%$ FBS for the indicated time. The cell lysate was applied to the kinase assay for ERK and immunoblots for phospho-ERK and ERK. ( $\boldsymbol{b}$ ) Densitometric measurements of phospho-ERK (ERK-1 and ERK-2) at baseline and $15 \mathrm{~min}$ after serum stimulation. The values are expressed as mean \pm SEM of four different pair of culture lines. ERK, extracellular signal-regulated kinase; $M B P$, myelin basic protein.

SDS-PAGE, electroblotted onto nitrocellulose membrane, and immunoblotted with phospho-specific p44/42 MAP kinase polyclonal antibody (New England Biolabs Inc., Beverly, Massachusetts, USA) or anti-ERK-1/2 antibody (Upstate Biotechnology, Inc.). Antibodies were detected by horseradish peroxidase-linked secondary antibody using an enhanced chemiluminescence system (Amersham Life Sciences Inc., Arlington Heights, Illinois, USA). Densitometric analysis was performed using an image scanner (Arcus II; Agfa Graphic Systems, Wilmington, Massachusetts, USA) and National Institutes of Health (Bethesda, Maryland, USA) image software. Densities of the bands corresponding to ERK-1 and ERK-2 were added to represent total ERK.

Effects of ERK kinase inhibitor on growth and ERK phosphorylation. The same pair of Agtr2 ${ }^{+}$and Agtr2- culture lines (E20) were used for DNA synthesis and phospho-ERK immunoblotting. Subconfluent, quiescent cells were treated with $10 \%$ FBS plus 0-100 $\mu \mathrm{M}$ ERK kinase (MEK) inhibitor PD98059 (New England Biolabs Inc.). DNA synthesis, $20 \mathrm{~h}$ stimulation followed by $4 \mathrm{~h}$ pulse labeling, was assayed as described above. ERK phosphorylation was measured after treating for $15 \mathrm{~min}$.

Determination of ERK dephosphorylation and MKP-1 level. ERK dephosphorylation was assayed according to the method described previously (11), with some modification. Sample lysates were prepared from subconfluent, quiescent cells (E20) in lysis buffer (20 mM HEPES [pH 7.5], 0.27 M sucrose, $2 \mathrm{mM}$ EDTA, 2 mM EGTA, 1\% Triton X-100, 1 mM $\beta$-glycerophosphate, $0.1 \% 2$-mercaptoethanol, $1 \mathrm{mM}$ benzamidine, $1 \mathrm{mM}$ PMSF, $10 \mu \mathrm{g} / \mathrm{ml}$ aprotinin). The lysates $(100 \mu \mathrm{g})$ were precipitated with the anti-ERK-1/2 antibody $(5 \mu \mathrm{g})$ to remove endogenous ERK. Consequently, the lysate supernatants were used for the dephosphorylation assay. Activated/phosphorylated ERK was immunoprecipitated with the anti-ERK-1/2 antibody using lysates of serum-stimulated Agtr2+ VSMC (E20). It was then incubated in dephosphorylation buffer $(40 \mathrm{mM}$ HEPES [pH 7.5], $0.01 \%$ BSA, $2 \mathrm{mM}$ dithiothreitol) for $1 \mathrm{~h}$ at room temperature with or without the sample lysates. ERK phosphorylation was detected by immunoblotting with phospho-specific p 44/42 MAP kinase monoclonal antibody (New England Biolabs Inc.). The density of the band was compared with that treated without the sample lysate; then, dephosphorylation was calculated as percent decrease in ERK phosphorylation. Protein levels of MKP-1 in the lysates were determined by immunoblotting using the anti-MKP-1 antibody (Santa Cruz Biotechnology Inc., Santa Cruz, California, USA).
Effects of vanadate and okadaic acid on ERK phosphorylation. Subconfluent VSMC (E20) were treated with the tyrosine phosphatase inhibitor vanadate $(0-20 \mu \mathrm{M}$; Sigma Chemical Co.) or with the serine/threonine phosphatase inhibitor okadaic acid (0 or $100 \mathrm{nM}$; Life Technologies Inc.), in serum-free DMEM for $16 \mathrm{~h}$. Phosphorylation and protein level of ERK were determined as described above.

Data analysis. The values in the text and figures are expressed as mean \pm SEM. The data were analyzed using one-factor ANOVA. If a statistically significant effect was found, NewmanKeuls' test was performed to isolate the difference between the groups. In some experiments using several different pair of Agtr2 ${ }^{+}$and Agtr2- culture lines, paired $t$ tests were also performed to clarify the difference, which might be invisible because of the variance among the culture lines. In interpreting results, $P<0.05$ was considered to be statistically significant.

\section{Results}

Developmental change of Ang II receptors and growth in VSMC. All of our culture lines (fetal through postnatal day 28) exhibited the "hill-and-valley" appearance characteristic of adult VSMC. The cells were uniformly positive for $\alpha$ smooth muscle actin by immunofluorescence. These results indicate that the cells are characteristic of VSMC, although the possible contamination of fibroblasts and endothelial cells cannot be avoided.

AT2 receptor expression in rat vasculature is reported to be abundant at the late gestational age and to decrease rapidly after birth $(23,24)$. Consistent with these results, reverse transcription-PCR analysis using mouse aorta revealed that AT2 receptor started to increase at E15, peaked at E20, and disappeared after seven days, whereas AT1 receptor was constantly expressed during the development (27). To examine whether the cultured VSMC retain the expression pattern for Ang II receptors, we determined AT1 and AT2 receptor expression by receptor binding assay. In Agtr2 ${ }^{+}$VSMC, AT2 receptor binding density increased by age, peaking at E20, and dramatically decreased after birth (Fig. 1), whereas Agtr2VSMC did not show any significant AT2 receptor binding (data not shown). In contrast, AT1 receptor binding density did not change developmentally or did not show 
difference between Agtr2+ and Agtr2- (Fig. 1). AT2 receptor binding density in Agtr2 $2^{+}$cells was significantly lower than AT1 binding density at each age (one-third of the AT1 receptor binding at E20).

By using these cells, we examined the developmental change in VSMC growth. The rate of growth, determined by the cell number increase in response to $10 \%$ fetal bovine serum (FBS) for four days, decreased according to age (Fig. 2). Importantly, VSMC growth at E18, E20, and postnatal day 7 was higher in Agtr2- than in Agtr2+, inversely correlating with the presence of the AT2 receptor. No difference between Agtr2 ${ }^{+}$and Agtr2- was observed at E15 or postnatal day 28, when the AT2 receptor is minimally or not expressed. Thus, developmental decline in VSMC growth was delayed in cells derived from Agtr2- mice.

Ang II-dependent and-independent growth of VSMC. To examine the mechanism of the growth difference, we studied VSMC prepared from E20 fetuses (which express abundant vascular AT2 receptor) and used these cells in the following experiments. The growth difference in response to $10 \%$ FBS between Agtr2 ${ }^{+}$and Agtr2- VSMC persisted for up to eight days of culture at the second passage (data not shown). Third-passage cells exhibited a growth phenotype similar to second-passage cells, while fourth-passage cells lost the growth ability and the morphological characteristics of VSMC (data not shown), suggesting that these cells reach terminal senescence at the fourth passage.

Because Agtr2 ${ }^{+}$VSMC express the significant amount of AT2 receptor, and we have reported that the AT2 receptor mediates growth inhibition in $\operatorname{VSMC}(4,5)$, we investigated whether the growth difference between Agtr2 ${ }^{+}$and Agtr2- VSMC was Ang II-dependent or-independent. Second-passage cultured VSMC were stimulated with Ang II or 10\% FBS, and the effects of Ang II receptor blockers were examined (Fig. 3, $a$ and $b$ ). In Agtr2 ${ }^{+}$VSMC, Ang II did not increase the cell number, but Ang II plus AT2 blocker PD123319 enhanced the growth, suggesting that the AT1 receptor stimulates cell growth and the AT2 receptor antagonizes it. Consistent with this, we observed that Ang II stimulated cell growth in Agtr2- VSMC. Interestingly, significant growth difference between Agtr2 ${ }^{+}$and Agtr2- VSMC was found even in the absence of Ang II and 10\% FBS (Fig. 3 , vehicle). Moreover, the growth difference in response to $10 \%$ FBS was not affected by AT2 blocker in either the Agtr2 ${ }^{+}$or Agtr2- cells (Fig. 3b), indicating that this difference did not depend on a direct activation of the cellsurface AT2 receptor by its peptide ligand, but rather by a possible programming effect on growth response by the AT2 receptor in vivo during development.

The effects of growth factors on DNA synthesis in VSMC (E20) were examined. DNA synthesis in secondpassage Agtr2- VSMC was also higher than that in corresponding Agtr2 ${ }^{+}$VSMC, in response to $10 \%$ FBS, PDGF-BB, and bFGF, as well as vehicle (Fig. 3c), supporting the notion that AT2 receptor null cells have the exaggerated growth phenotype.

ERK and its link with the growth phenotype. It is well known that ERK plays a pivotal role in regulating cell growth (16). Previous studies have shown that AT2 receptor stimulation inactivates ERK via the activation of tyrosine

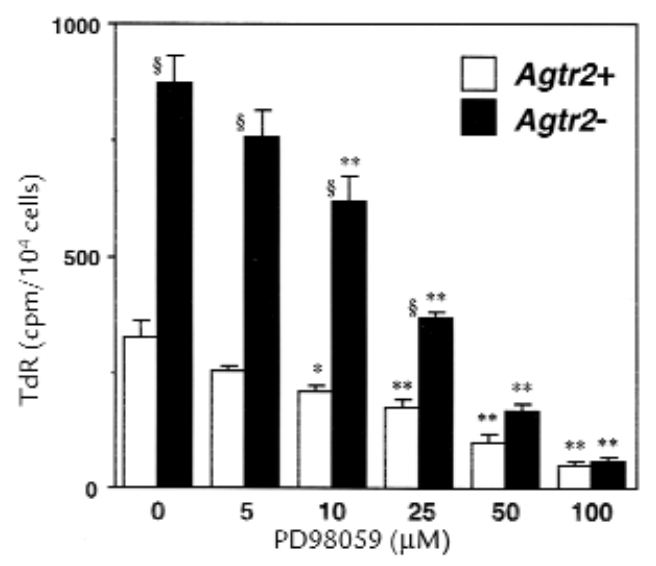

$b$

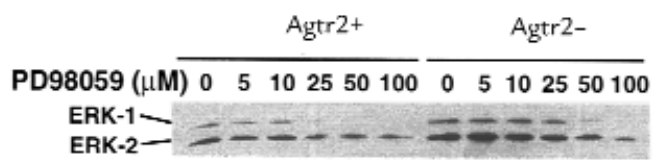

$c$
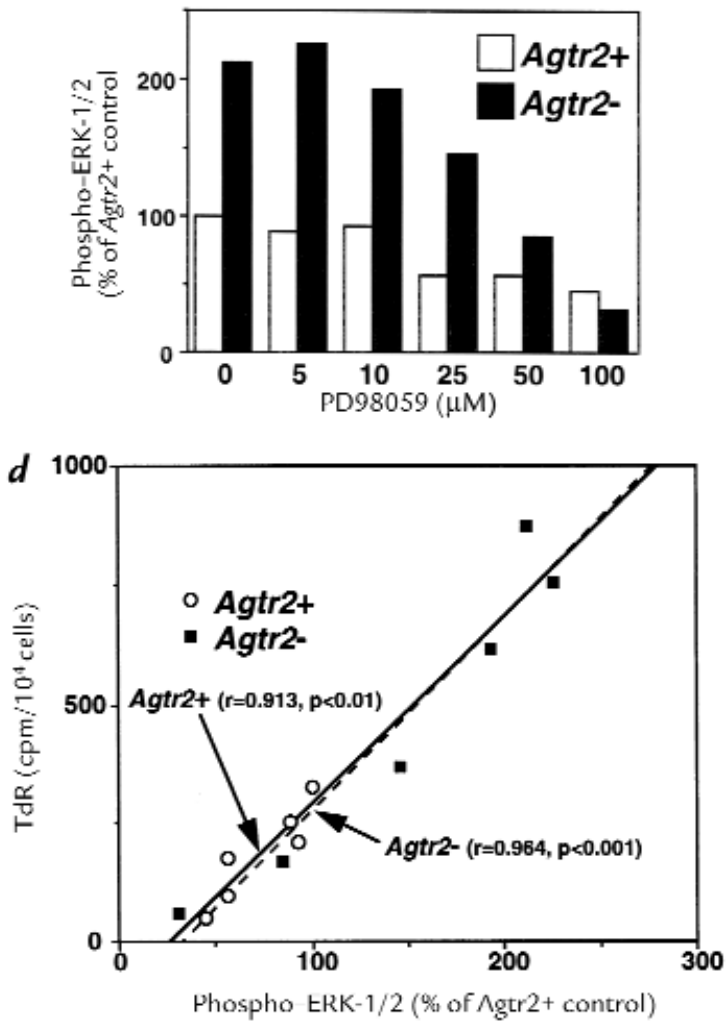

Figure 5

Effects of MEK inhibitor on DNA synthesis and ERK phosphorylation in wild-type $\left(\right.$ Agtr2 $\left.2^{+}\right)$and AT2 receptor null (Agtr2-) VSMC derived from the aortae of embryonic day 20 fetuses. Subconfluent, quiescent cells were treated with 10\% FBS plus 0-100 $\mu \mathrm{M}$ MEK inhibitor PD98059. (a) DNA synthesis was assayed as ${ }^{3} \mathrm{H}$-thymidine incorporation $(T d R)$. The values are expressed as mean $\pm \operatorname{SEM}(n=6)$. ${ }^{*},{ }^{*} P<0.05,0.01$ vs. PD98059 $(-) ; § P<0.05$ vs. Agtr2+. (b and $\boldsymbol{c})$ Immunoblotting for phospho-ERK and its densitometric analysis are shown. (d) Scatter diagram showing the correlation between DNA synthesis and ERK phosphorylation. The regression lines are identical in Agtr2 ${ }^{+}$and Agtr2-. MEK, ERK kinase. 


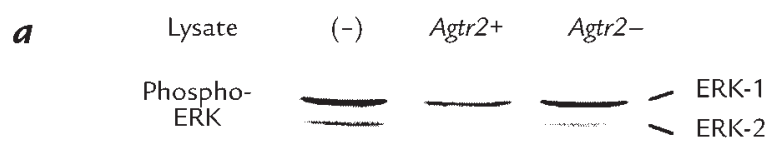

Decrease in ERK Phosphorylation

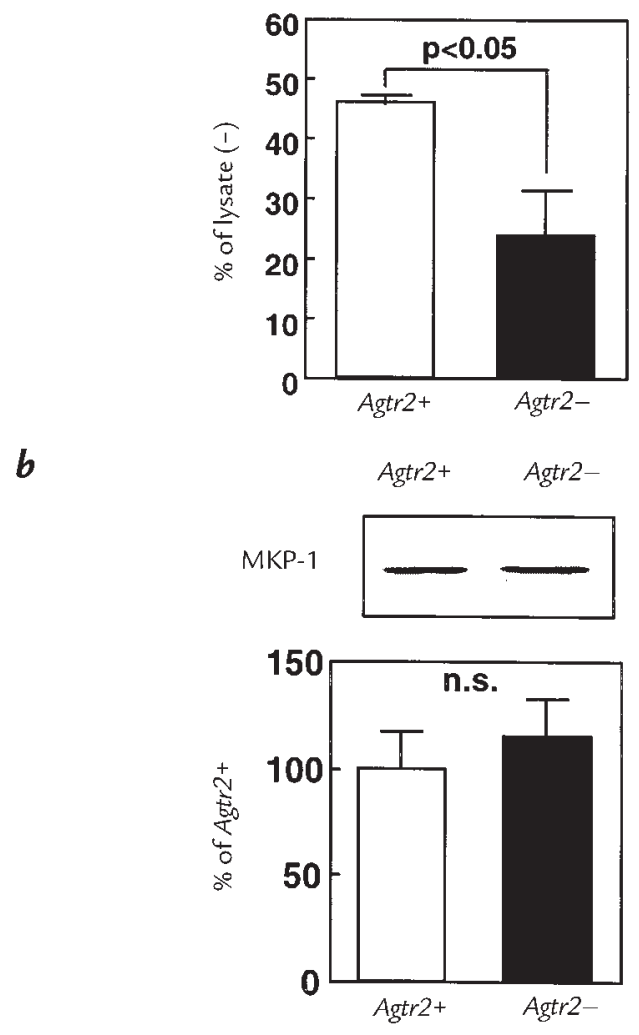

Figure 6

ERK dephosphorylation and MKP-1 protein level in wild-type $\left(\right.$ Agtr2 $\left.{ }^{+}\right)$and AT2 receptor null (Agtr2-) VSMC. (a) Phosphorylated ERK was incubated with the lysates prepared from Agtr2 ${ }^{+}$and Agtr2- VSMC and was detected by immunoblotting. The density of the band was compared with that treated without the sample lysate; then, dephosphorylation was calculated as percent decrease in ERK phosphorylation. (b) Protein levels of MKP-1 in the lysates were determined by immunoblotting using the anti-MKP-1 antibody. The values are expressed as mean \pm SEM of four different pair of culture lines. MKP-1, MAP kinase phosphatase-1.

phosphatases $(5,11,12,17)$. Consistent with this, we have demonstrated the attenuated ERK activity in vivo in AT2 receptor in transgenic mouse hearts (28). Therefore, we hypothesized that ERK activity would be higher in AT2 receptor null VSMC, resulting in a higher growth rate compared with Agtr2 ${ }^{+}$cells.

As shown in Fig. 4a, both kinase assay and immunoblot using anti-phospho-ERK antibody documented that ERK activity was higher in Agtr2- VSMC at baseline (before serum stimulation) and also in response to $10 \%$ FBS, whereas the protein levels of ERK were similar. Because densitometric measurements of immunoblotting for phospho-ERK correlated very well with ERK activities determined by kinase assay $(r=0.979, P<0.001$, $n=8$ ), we used phospho-ERK immunoblotting to examine the ERK activity in the following experiments. Den- sitometric analysis using four different pair of culture lines showed that phospho-ERK was $200 \%$ higher at baseline and $140 \%$ higher 15 minutes after serum stimulation in Agtr2- than in Agtr2 $2^{+}$VSMC (Fig. 4b). In contrast, VSMC from E15 fetuses or postnatal day 28 animals did not show the difference in ERK phosphorylation between Agtr2 $2^{+}$and Agtr2- (data not shown).

To examine whether ERK activity is linked to growth response, we treated the cells with MEK inhibitor PD98059. PD98059 inhibited both growth as measured by DNA synthesis and ERK phosphorylation, dose dependently (Fig. 5, $a-c$ ). Interestingly, the regression lines between VSMC thymidine incorporation and ERK phosphorylation were identical in Agtr2 ${ }^{+}$and Agtr2cells (Fig. 5d), suggesting that the increased growth in Agtr2- cells is attributable to a higher level of intracellular ERK activity.

Because it has been reported that protein tyrosine phosphatases play a critical role in AT2 receptor-mediated ERK inactivation $(5,11,12,17)$, we studied the involvement of phosphatases in ERK activity in these cells. We examined phosphatase activity that determined ERK activity in Agtr2 $2^{+}$and Agtr2- VSMC by measuring ERK dephosphorylation (Fig. 6a). The phosphatase activity of the Agtr2+ cells was approximately twofold higher than that of Agtr2- cells, showing a reasonable difference that would explain the difference in ERK activity. These results suggest that the activity of some specific phosphatase(s) that directly regulates ERK activity is enhanced in Agtr2- cells. Next, we examined the protein level of MKP-1, which is a dual specificity phosphatase that dephosphorylates ERK, and demonstrated that MKP-1 protein levels were similar in Agtr2 $2^{+}$and Agtr2- VSMC. As shown in Fig. 7, $a$ and $b$, vanadate increased ERK phosphorylation predominantly in Agtr2 ${ }^{+}$cells and abolished the difference between Agtr2 $2^{+}$and Agtr2- cells. In contrast, $100 \mathrm{nM}$ of okadaic acid, a concentration enough to inhibit protein phosphatases 1 and 2A (29) and exhibit a small increase in ERK phosphorylation in both cells, did not influence the difference between Agtr2 $2^{+}$and Agtr2- cells. The protein levels of ERK were not affected by the treatments. These results suggest that tyrosine phosphatase, rather than serine/threonine phosphatase, is a primary determinant in the quantitative difference of ERK activities between the Agtr2+ and Agtr2- VSMC.

\section{Discussion}

The adult intact artery, which is fully developed and differentiated, is a quiescent tissue. Indeed, replication rate of VSMC in the normal aorta is less than $0.05 \%$ per day (30). In contrast, VSMC in the developing aorta undergo remarkable proliferation (31-33). However, this replication rate exhibits a dramatic reduction in the late gestational stage $(4,33)$, when the AT2 receptor is abundantly expressed $(23,24)$. We have demonstrated that in utero pharmacologic blockade of the fetal AT2 receptor attenuated this decline in the rate of aortic DNA synthesis (4), indicating that the AT2 receptor modulates growth of the developing blood vessel and thus contributes to vascular remodeling in late gestation. The AT2 receptor null mouse provides us with an opportunity to further study 


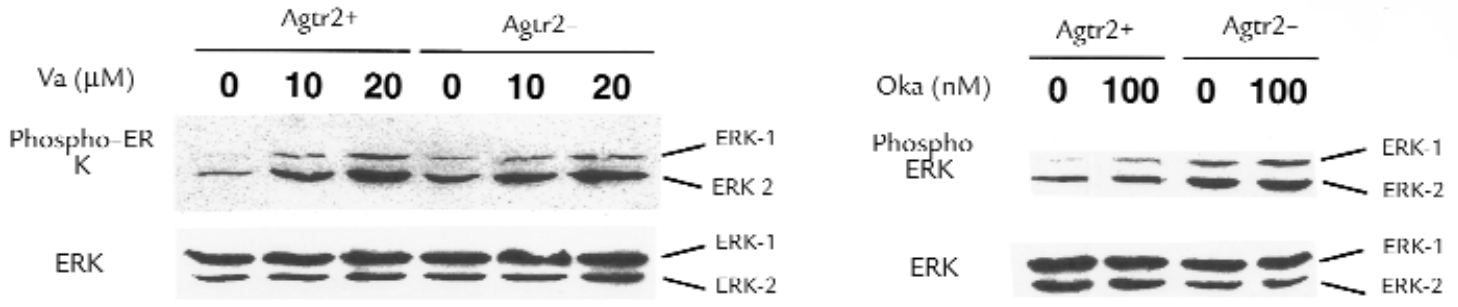

\section{$\boldsymbol{b}$}
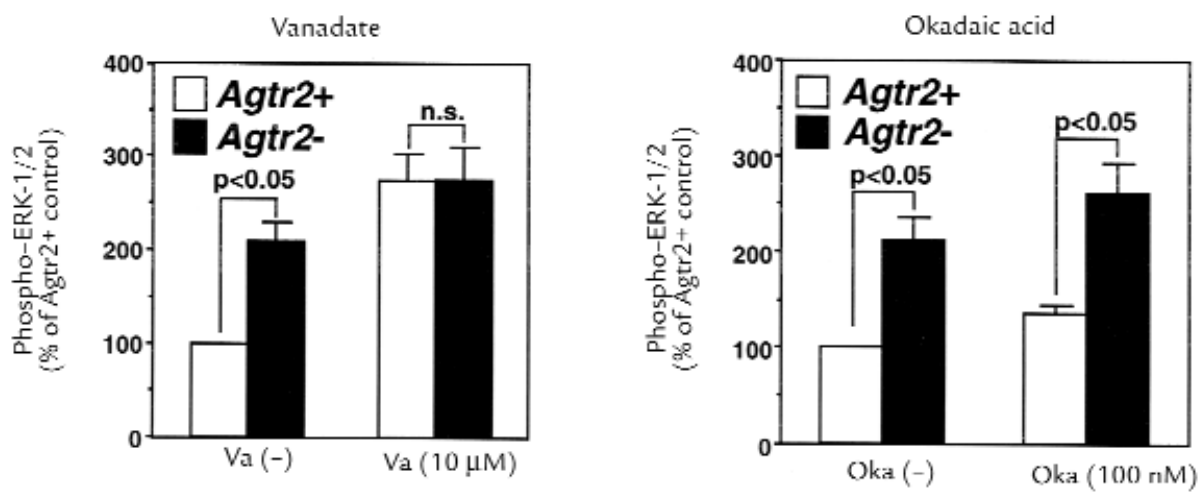

Figure 7

Effects of vanadate and okadaic acid on ERK phosphorylation in wild-type (Agtr2 $2^{+}$) and AT2 receptor null (Agtr2-) VSMC derived from the aortae of embryonic day 20 fetuses. Subconfluent VSMC were treated with vanadate (Va; 0-20 $\mu$ M) or okadaic acid (Oka; 0 or $100 \mathrm{nM})$ in serum-free medium for 16 h. (a) Immunoblotting for phospho-ERK and ERK. (b) The densitometric analysis for phospho-ERK. The values are expressed as mean \pm SEM of five (vanadate) or four (okadaic acid) different pair of culture lines.

the role of this receptor in vascular development. The AT2 receptor null mouse exhibits no gross abnormalities; however, the basal blood pressure and the acute response to Ang II are enhanced in the adult (three to five months old) AT2 receptor null mouse $(25,34)$. Because the vascular AT2 receptor is not expressed at this age, the data suggest that the transient and developmentally regulated AT2 receptor expression in late gestation exerts a long-term effect on blood pressure, possibly via its influence on vascular structure. However, the structural consequences of fetal AT2 receptor expression in vascular development in the AT2 receptor null mouse await detailed analysis.

To study the influence of AT2 receptor expression on the developmental biology of VSMC, we studied cultures of VSMC derived from wild-type and AT2 receptor null mice. In this study we demonstrated that VSMC derived from the aorta of fetal AT2 receptor null mice exhibited the exaggerated growth phenotype compared with agematched wild-type VSMC. ERK activity, which was closely associated with growth, was programmed to be higher in AT2 receptor null VSMC. This high ERK activity in AT2 receptor null cells may be attributable to the low tyrosine phosphatase activity.

Cook et al. (33) reported that cultured VSMC derived from rat aorta exhibited the developmentally regulated growth, mimicking the in vivo pattern. They showed that the high growth potential of fetal VSMC was lost by E20. These data suggest that the significant change in VSMC phenotype occurs during the development and that cul- tured VSMC reflect the in vivo phenotype. Consistent with their data, we observed that the growth rate of wild-type VSMC declined after E18. In contrast, the growth rate of AT2 receptor null VSMC remained increased up to postnatal day 7. These results suggest that AT2 receptor expression during the fetal vasculogenesis influences the growth phenotype of VSMC. However, the finding that the growth rate decreased developmentally, even in AT2 receptor null VSMC and in postnatal wild-type VSMC in which AT2 receptor was minimally expressed, indicates that some other factors are also implicated in this process. Little is known about the factors that control the growth phenotype of VSMC during development. Majesky et al. $(35,36)$ have reported that developmentally regulated expression of endogenous PDGF and its receptor influences rat VSMC growth. In our preliminary study, however, conditioned medium derived from cultured AT2 receptor null VSMC did not contain higher growth-promoting activity than that from wild-type VSMC (data not shown). Furthermore, AT2 receptor null cells showed greater growth responses to serum, PDGF, and bFGF, indicating that AT2 receptor null VSMC exhibit a generalized exaggerated growth phenotype. Thus, an alteration in signaling pathways in AT2 receptor null VSMC is likely to be responsible for the exaggerated growth phenotype, rather than alterations in growth factors or their receptor expressions.

In this study, we focused on the ERK pathway because this cascade is crucial in proliferation, differentiation, and hypertrophy of VSMC (16), and is implicated in the AT2 
receptor-mediated antigrowth and proapoptotic actions on $\operatorname{VSMC}(4,5,10)$. ERK is also involved in migration (37) and contraction $(38,39)$ of VSMC, denoting that ERK plays pivotal roles in the regulation of VSMC biology. We observed that ERK activity was significantly higher in AT2 receptor null VSMC than in wild-type VSMC at baseline and in response to serum, whereas protein levels of ERK were similar, suggesting that the difference is due to activation/phosphorylation, not to transcription or the translation of ERK. We demonstrated that phosphatase activity, which directly regulated ERK, was higher in wild-type VSMC than in AT2 receptor null VSMC.or vanadate, but not serine/threonine phosphatase inhibitor okadaic acid, abolished the difference in the basal ERK phosphorylation between wild-type and AT2 receptor null VSMC without influence on ERK protein levels supports the notion that tyrosine phosphatases are involved in programming the level of ERK activity in these cells. To investigate the upstream events in the ERK cascade, we determined MEK phosphorylation in the cells. We could not detect a significant difference in MEK phosphorylation between wild-type and AT2 receptor null VSMC (data not shown), suggesting that the kinase activity in AT2 receptor null cells is increased at the level of ERK. However, possible regulation of other signaling molecules by decreased tyrosine phosphatase activities in AT2 receptor null VSMC still remains to be defined. Some tyrosine phosphatases are shown to be critical to embryonic development (40-42) and regulation of VSMC function $(43,44)$. Tyrosine phosphatases can regulate the development and function of VSMC by dephosphorylating their target substrates, including ERK. Given that stimulation of the AT2 receptor activates tyrosine phosphatases in vivo, the precise mechanism by which multiple-passaged cultured VSMC maintain this phenotype in vitro in the absence of Ang II, has to be explained. One possible mechanism is developmental programming. We hypothesize that the developmental expression and activation of vascular AT2 receptor stimulate tyrosine phosphatase activity, whereas the lack of this receptor in AT2 receptor null vessels results in lower enzyme activity. This developmental regulation programs the subsequent level of local tyrosine phosphatase activity even in the absence of AT2 receptor activation or expression. Indeed, the finding that cultured VSMC derived from genetic disease animals such as spontaneously hypertensive rats (45) and spontaneously diabetic rats (46) exhibit the exaggerated growth phenotype supports this possibility. The exact molecular mechanism of genetic programming of intracellular regulatory pathways is currently unknown.

The results of this study may be relevant to vascular pathobiology. It has been shown that neointimal VSMC in vascular lesions such as atherosclerosis and restenosis after balloon angioplasty resemble fetal VSMC, rather than adult medial VSMC, in terms of morphology, expression of smooth-muscle specific markers, and growth ability $(36,47)$. Furthermore, ERK is activated in neointimal VSMC (48), and the downregulation of MKP-1 is associated with the activation of ERK after vascular injury (44). Our data may provide an explanation for why these altered growth phenotypes persist in culture. We hypothesize that in vivo expression and activation or inactivation of specific growth regulatory receptors and their signaling pathways for a specific time period can have a genetic programming effect that subsequently influences the phenotype, at least for several cell cycles. In the case of AT2 receptor, it is reexpressed in injured blood vessels and attenuates neointima formation $(4,20)$. The expression pattern may play a role in the reversal of the phenotypic shift, thereby participating in the vascular remodeling in these pathophysiologic states.

\section{Acknowledgments}

This work was supported by National Institutes of Health (NIH) grants HL-46631, HL-35252, HL-35610, HL-48638, HL07708, and HL-58616, and by a grant from the Longwood Foundation for Translational Research. V.J. Dzau is a recipient of NIH Merit Award HL-35610.

1. Dzau, V.J., Gibbons, G.H., and Pratt, R.E. 1991. Molecular mechanisms of vascular renin-angiotensin system in myointimal hyperplasia. Hypertension. 18(Suppl II):100-105.

2. Mukoyama, M., et al. 1993. Expression cloning of type 2 angiotensin II receptor reveals a unique class of seven-transmembrane receptors. J. Biol. Chem. 268:24539-24542.

3. Kambayashi, Y., et al. 1993. Molecular cloning of novel angiotensin II receptor isoform involved in phosphotyrosine phosphatase inhibition. J. Biol. Chem. 268:24543-24546.

4. Nakajima, M., et al. 1995 . The angiotensin II type 2 (AT2) receptor antagonizes the growth effects of the AT1 receptor: gain of function study using in vivo gene transfer. Proc. Natl. Acad. Sci. USA. 92:10663-10667.

5. Hayashida, W., Horiuchi, M., and Dzau, V.J. 1996. Intracellular third loop domain of angiotensin II type 2 receptor: role in mediating signal transduction and cellular function. J. Biol. Chem. 271:21985-21992.

6. Stoll, M., et al. 1995. The angiotensin AT2-receptor mediates inhibition of cell proliferation in coronary endothelial cells. J. Clin. Invest. 95:651-657.

7. Meffert, S., Stoll, M., Steckelings, U.M., Bottari, S.P., and Unger, T. 1996. The angiotensin II AT2 receptor inhibits proliferation and promotes differentiation in PC12W cells. Mol. Cell. Endocrinol. 122:59-67.

8. Booz, G.W., and Baker, K.M. 1996. Role of type 1 and type 2 angiotensin receptors in angiotensin II-induced cardiomyocyte hypertrophy. Hypertension. 28:635-640.

9. Laflamme, L., Gasparo, M., Gallo, J.M., Payet, M.D., and Gallo-Payet, N. 1996. Angiotensin II induction of neurite outgrowth by AT2 receptors in NG108-15 cells. Effect counteracted by the AT1 receptors. J. Biol. Chem. 271:22729-22735

10. Yamada, T., et al. 1998. Angiotensin II type 2 receptor mediates vascular smooth muscle cell apoptosis and antagonizes angiotensin II type 1 receptor action: an in vitro gene transfer study. Life Sci. 63:PL289-PL295.

11. Yamada, T., Horiuchi, M., and Dzau, V.J. 1996. Angiotensin II type 2 receptor mediates programmed cell death. Proc. Natl. Acad. Sci. USA. 93:156-160.

12. Horiuchi, M., Hayashida, W., Kambe, T., Yamada, T., and Dzau, V.J. 1997. Angiotensin II type 2 receptor dephosphorylates $\mathrm{Bcl}-2$ by activating mitogen-activated protein kinase phosphatase- 1 and induces apoptosis. J. Biol. Chem. 272:19022-19026.

13. Hayashida, W., Horiuchi, M., Grandchamp, J., and Dzau, V.J. 1996. Antagonistic action of angiotensin II type- 1 and type- 2 receptors on apoptosis in cultured neonatal rat ventricular myocytes. Hypertension. 28:535. (Abstr.)

14. Horiuchi, M., Yamada, T., Hayashida, W., and Dzau, V.J. 1997. Interferon regulatory factor- 1 upregulates angiotensin type 2 receptor and induces apoptosis. J. Biol. Chem. 272:11952-11958.

15. Dimmeler, S., Rippmann, V., Weiland, U., Haendeler, J., and Zeiher, A.M. 1997. Angiotensin II induces apoptosis of human endothelial cells. Protective effect of nitric oxide. Circ. Res. 81:970-976.

16. Force, T., and Bonventre, J.V. 1998. Growth factors and mitogen-activated protein kinases. Hypertension. 31:152-161.

17. Bedecs, K., et al. 1997. Angiotensin II type 2 receptors mediate inhibition of mitogen-activated protein kinase cascade and functional activation of SHP-1 tyrosine phosphatase. Biochem. J. 325:449-454.

18. Daud, A.I., Bumpus, F.M., and Husain, A. 1988. Evidence for selective expression of angiotensin II receptors on atretic follicles in the rat ovary: an autoradiographic study. Endocrinology. 122:2727-2734.

19. Grady, E.F., Sechi, L.A., Griffin, C.A., Schambelan, M., and Kalinyak, J.E. 1991. Expression of AT2 receptors in the developing rat fetus. J. Clin. Invest. 88:921-933. 
20. Akishita, M., Horiuchi, M., Yamada, H., Zhang, L., and Dzau, V.J. 1997. Accentuated vascular proliferation and altered remodeling after injury in mice lacking angiotensin II type 2 receptor. Circulation. 96:1-547. (Abstr.)

21. Nio, Y., Matsubara, H., Murasawa, S., Kanasaki, M., and Inada, M. 1995. Regulation of gene transcription of angiotensin II receptor subtypes in myocardial infarction. J. Clin. Invest. 95:46-54.

22. Wharton, J., et al. 1998. Differential distribution of angiotensin AT2 receptors in the normal and failing human heart. J. Pharmacol. Exp. Ther 284:323-336.

23. Viswanathan, M., Tsutsumi, K., Correa, F.M., and Saavedra, J.M. 1991. Changes in expression of angiotensin receptor subtypes in the rat aorta during development. Biochem. Biophys. Res. Commun. 179:1361-1367.

24. Shanmugam, S., Corvol, P., and Gasc, J-M. 1996. Angiotensin II type 2 receptor mRNA expression in the developing cardiopulmonary system of the rat. Hypertension. 28:91-97.

25. Hein, L., Barsh, G.S., Pratt, R.E., Dzau, V.J., and Kobilka, B.K. 1995. Behavioural and cardiovascular effects of disrupting the angiotensin II type-2 receptor gene in mice. Nature. 377:744-747.

26. Skalli, O., et al. 1986. A monoclonal antibody against $\alpha$-smooth muscle actin: a new probe for smooth muscle differentiation. J. Cell Biol. 103:2787-2796.

27. Yamada, H., Horiuchi, M., Akishita, M., and Dzau, V.J. 1997. Regulation of vascular development and differentiation by angiotensin II type 2 receptor. Hypertension. 30:470. (Abstr.)

28. Masaki, H., et al. 1998. Cardiac-specific overexpression of angiotensin II AT2 receptor causes attenuated response to AT1 receptor-mediated pressor and chronotropic effects. J. Clin. Invest. 101:527-535.

29. Cohen, P., Holmes, C.F., and Tsukitani, Y. 1990. Okadaic acid: a new probe for the study of cellular regulation. Trends Biochem. Sci. 15:98-102.

30. Lombardi, D.M., Reidy, M.A., and Schwartz, S.M. 1991. Methodologic considerations important in the accurate quantitation of aortic smooth muscle cell replication in the normal rat. Am. J. Pathol. 138:441-446.

31. Owens, G.K., and Thompson, M.M. 1986. Developmental changes in isoactin expression in rat aortic smooth muscle cells in vivo. Relationship between growth and cytodifferentiation. J. Biol. Chem. 261:13373-13380.

32. Bochaton-Piallat, M.L., Gabbiani, F., Ropraz, P., and Gabbiani, G. 1993. Age influences the replicative activity and the differentiation features of cultured rat aortic smooth muscle cell populations and clones. Arterioscler. Thromb. 13:1449-1455.

33. Cook, C.L., Weiser, M.C., Schwartz, P.E., Jones, C.L., and Majack, R.A 1994. Developmentally timed expression of an embryonic growth phenotype in vascular smooth muscle cells. Circ. Res. 74:189-196.

34. Ichiki, T., et al. 1995. Effects on blood pressure and exploratory behav- iour of mice lacking angiotensin II type-2 receptor. Nature. 377:748-750.

35. Majesky, M.W., Benditt, E.P., and Schwartz, S.M. 1988. Expression and developmental control of platelet-derived growth factor A-chain and Bchain/Sis genes in rat aortic smooth muscle cells. Proc. Natl. Acad. Sci. USA. 85:1524-1528.

36. Majesky, M.W., Giachelli, C.M., Reidy, M.A., and Schwartz, S.M. 1992. Rat carotid neointimal smooth muscle cells reexpress a developmentally regulated mRNA phenotype during repair of arterial injury. Circ. Res. 71:759-768.

37. Graf, K., et al. 1997. Mitogen-activated protein kinase activation is involved in platelet-derived growth factor-directed migration by vascular smooth muscle cells. Hypertension. 29:334-339.

38. Khalil, R.A., Menice, C.B., Wang, C.L., and Morgan, K.G. 1995. Phosphotyrosine-dependent targeting of mitogen-activated protein kinase in differentiated contractile vascular cells. Circ. Res. 76:1101-1108.

39. Epstein, A.M., Throckmorton, D., and Brophy, C.M. 1997. Mitogen-activated protein kinase activation: an alternate signaling pathway for sustained vascular smooth muscle contraction. J. Vasc. Surg. 26:327-332.

40. Tang, T.L., Freeman, R.M. Jr., O’Reilly, A.M., Neel, B.G., and Sokol, S.Y. 1995. The SH2-containing protein-tyrosine phosphatase SH-PTP2 is required upstream of MAP kinase for early Xenopus development. Cell. 80:473-483.

41. Saxton, T.M., et al. Abnormal mesoderm patterning in mouse embryos mutant for the SH2 tyrosine phosphatase Shp-2. EMBO J. 16:2352-2364.

42. Fuchs, M., Wang, H., Ciossek, T., Chen, Z, and Ullrich, A. 1998. Differential expression of MAM-subfamily protein tyrosine phosphatases during mouse development. Mech. Dev. 70:91-109.

43. Duff, J.L., et al. 1993. Angiotensin II induces 3CH134, a protein-tyrosine phosphatase, in vascular smooth muscle cells. J. Biol. Chem. 268:26037-26040.

44. Lai, K., et al. 1996. Mitogen-activated protein kinase phosphatase-1 in rat arterial smooth muscle cell proliferation. J. Clin. Invest. 98:1560-1567.

45. Hadrava, V., et al. 1991. Vascular smooth muscle cell proliferation and its therapeutic modulation in hypertension. Am. Heart J. 122:1198-1203.

46. Yoo, H.J., et al. 1997. Augmented Ca2+ influx is involved in the mechanism of enhanced proliferation of cultured vascular smooth muscle cells from spontaneously diabetic Goto-Kakizaki rats. Atherosclerosis. 131:167-175.

47. Schwartz, S.M., Campbell, G.R., and Campbell, J.H. 1986. Replication of smooth muscle cells in vascular disease. Circ. Res. 58:427-444.

48. Hu, Y., Cheng, L., Hochleitner, B.W., and Xu, Q. 1997. Activation of mitogen-activated protein kinases (ERK/JNK) and AP-1 transcription factor in rat carotid arteries after balloon injury. Arterioscler. Thromb. Vasc. Biol. 17:2808-2816. 